\title{
Les politiques linguistiques françaises par rapport au bilinguisme précoce: quelles leçons en tirer pour le Mexique?
}

\author{
Haydée SILVA OCHOA \\ Universidad Nacional Autónoma de México
}

Dresser en quelques pages un panorama détaillé des politiques linguistiques européennes et mexicaine relatives à l'enseignement/apprentissage d'une langue étrangère avant l'âge de 11-12 ans est tout simplement impossible. Le parcours propósé ici inclut donc quatre courtes étapes: nous aborderons dans un premier temps les grandes lignes des politiques linguistiques européennes actuelles, pour ensuite nous intéresser de plus près à la situation spécifique de la France. Puis nous évoquerons l'écart entre politiques officielles et réalités sur le terrain. Enfin, nous nous interrogerons sur ce que la connaissance des politiques linguistiques européennes peut nous apporter au Mexique.

1. Les grands axes des politiques linguistiques européennes relatives au bilinguisme et à l'enseignement/apprentissage précoce des langues vivantes

En effet, depuis 15 ans, les politiques linguistiques européennes, définies par le Conseil de l'Europe, ont subi des transformations radicales. Pour en rendre brièvement compte, nous allons faire principalement mais non exclusivement appel à deux sources fort complètes, le Cadre Européen Commun de Référence (Conseil de l'Europe, 2001) et L'Enseignement des langues étrangères en milieu scolaire en Europe (Eurydice, 2001), respectivement appelées à partir de maintenant "Cadre" et "Eurydice". Nous avons également obtenu des données plus récentes sur divers sites électroniques ainsi qu'à partir du Monde de l'éducation.

Quelle est la place accordée par le Cadre à la problématique du bilinguisme? En tant que tel, celui-ci mérite à peine quelques mentions rapides. Néanmoins, le Cadre évoque à plusieurs reprises l'importance d'une compétence "plurilingue et pluriculturelle". La définition de cette compétence diffère explicitement du bilinguisme et permet de saisir quel est l'angle d'approche choisi en Europe par rapport à la problématique qui nous occupe.

La compétence plurilingue et pluriculturelle est définie dans le Cadre comme 
[...] la compétence à communiquer langagièrement et à interagir culturellement d'un acteur social qui possède, à des degrés divers, la maîtrise de plusieurs langues et l'expérience de plusieurs cultures. [...] il n'y a pas là superposition ou juxtaposition de compétences distinctes, mais bien existence d'une compétence complexe, voire composite, dans laquelle l'utilisateur peut puiser. [...] La notion de compétence plurilingue et pluriculturelle tend à sortir de la dichotomie d'apparence équilibrée qu'instaure le couple habituel $\mathrm{L} 1 / \mathrm{L} 2$, en insistant sur le plurilinguisme dont le bilinguisme n'est qu'un cas particulier (c'est nous qui soulignons; Conseil de l'Europe, 2001: 129).

Sans entrer dans une discussion détaillée, on ne peut manquer d'observer que, de manière explicite, le débat n'est plus posé en termes de bilinguisme mais de plurilinguisme, et que la compétence correspondante va bien au-delà d'un savoir linguistique ou même d'une aptitude à la communication, car elle accorde une place primordiale au langage plutôt qu'à la langue; à l'interaction; à l'action sociale et à la culture. Le Cadre insiste aussi fortement sur la complexité du phénomène, qui n'est pas une simple superposition ou une juxtaposition de deux langues.

Le Cadre rappelle par ailleurs qu'en Europe, la langue de scolarisation dominante est loin d'être toujours la langue maternelle des enfants scolarisés. ' Cela revient à souligner que la problématique du plurilinguisme, profondément ancrée dans la réalité des pays européens, dépasse largement le domaine théorique de la planification curriculaire et même celui de la conception "en surplomb" des politiques linguistiques. Il est effectivement essentiel de rappeler que le bilinguisme ou le plurilinguisme ne concernent pas uniquement l'opposition langue nationale/langue(s) étrangère(s) mais qu'ils sont aussi et souvent avant tout affaire d'opposition langue nationale/langue(s) minoritaire(s). C'est un aspect important pour le Mexique, dans la mesure où il est clair que le bilinguisme ou le plurilinguisme ne concernent pas uniquement l'opposition langue nationale/langue(s) étrangère(s) mais qu'ils sont aussi et souvent avant tout affaire d'opposition langue nationale/langue(s) minoritaire(s).

Le Cadre apporte également divers éclaircissements relatifs aux compétences visées par l'enseignement scolaire des langues ainsi qu'aux deux scénarios curriculaires dominants. Cependant, il nous faut désormais aborder ici plus concrètement les orientations prises par les politiques linguistiques européennes, telles qu'elles sont présentées dans Eurydice. C'est un ouvrage particulièrement

\footnotetext{
${ }^{1}$ Eury dice abonde dans le même sens: "seuls seize des vingt-neuf pays étudiés possèdent une langue officielle unique, les autres en ayant entre deux et cinq. [... Seuls] deux pays (l'Islande et le Liechtenstein) peuvent être qualifiés de monolingues, puisqu'ils n'ont mentionné qu'une seule langue officielle et aucune langue minoritaire ou régionale" (Eurydice, 2001:21).
} 
intéressant, car il évoque au cas par cas tout autant l'organisation pédägogique de l'enseignement des langues étrangères en milieu scolaire que les approches et le contenu des programmes d'études, les mesures prises dans la formation et le recrutement des enseignants ainsi que les actions de soutien linguistique développées dans les systèmes éducatifs à l'égard des langues minoritaires ( $c f$. Eurydice, 2001: 3).

Les auteurs de cet ouvrage rappellent dans leur introduction comment, depuis 1995, la priorité donnée à l'acquisition de compétences linguistiques par tous les citoyens européens a été

[...] clairement affirmée dans le Livre Blanc de la Commission européenne sur l'éducation et la formation [...] au travers des objectifs suivants: "Développer l'enseignement d'au moins deux langues communautaires étrangères pour tous les jeunes; encourager les méthodes innovantes d'apprentissage des langues; diffuser la pratique quotidienne des langues étrangères européennes au sein des établissements scolaires de tous les niveaux; favoriser la sensibilisation aux langues et cultures communautaires, ainsi que l'apprentissage précoce de celles-ci" (Eurydice, 2001: 7; c'est nous qui soulignons).

En effet, la maîtrise d'au moins deux langues étrangères au terme de la scolarité devient un objectif clé, afin que "tous les citoyens puissent bénéficier pleinement de leur droit à la libre circulation et devenir acteurs d'une meilleure compréhension mutuelle entre les peuples européens" (Eurydice, 2001: 3). Pour atteindre cet objectif, les 29 pays étudiés par Eurydice

[...] ont modifié le statut de l'enseignement des langues étrangères, en assurant une présence obligatoire de cette matière à l'intérieur du programme et en s'efforçant de prolonger la durée de son enseignement (généralement en avançant l'âge à partir duquel la première langue étrangère est enseignée). [... Cependant, dans] la majorité des pays de l'Union, il faut attendre les années 80 , voire 90 , pour observer la mise en œuvre des réformes rendant la langue étrangère obligatoire dans le programme d'études (Eurydice, 2001: 60).

Il convient de préciser que ces remarques sont applicables uniquement au niveau primaire, car "Au niveau préprimaire, les expériences sont très récentes et ne concernent qu'un groupe très réduit de pays [Autriche, Espagne, Italie]" (Eurydice, 2001: 70).

C'est aussi en 1995 qu'une Résolution du Conseil "faisait le point sur les nouveaux pouvoirs accordés à la Communauté dans [le domaine de 
l'apprentissage et la diffusion des langues des États membres] et constatait que la promotion de la diversité linguistique était devenue l'un des enjeux principaux en matière d'éducation" (Eurydice, 2001: 174). Parmi les mesures à prendre, on faisait allusion notamment à: "la promotion de la mobilité des enseignants et des apprenants; la promotion des méthodes pédagogiques novatrices; l'apprentissage des langues dès le plus jeune âge; la définition des compétences des enseignants de langues; la nécessité pour les élèves d'apprendre deux langues différentes de leur langue maternelle durant au moins deux ans; la nécessité d'améliorer l'offre éducative en matière de langues moins diffusées et moins enseignées" (idem).

À notre sens, il est important de replacer le souci particulier relatif à l'apprentissage précoce parmi le reste des priorités établies par le Conseil de l'Europe, afin de mieux insister sur l'interrelation de la problématique du bilinguisme ou du plurilinguisme précoce avec d'autres facteurs essentiels qui ne concernent pas uniquement les enfants et ceux qui les entourent. La mobilité, le renouveau pédagogique, la formation linguistique, culturelle et pédagogique des enseignants et l'équilibre entre les diverses langues sont autant d'éléments qui concernent peu ou prou tous ceux qui s'intéressent au Français Langue Étrangère, au Mexique ou ailleurs.

Faute de place, nous ne pourrons développer l'intérêt porté par Eurydice aux orientations visant à favoriser l'intégration dont la langue maternelle est étrangère au sein d'un modèle d'enseignement qui se veut désormais "interculturel, axé sur le respect mutuel, l'éveil à d'autres cultures et les compétences transculturelles" ( $c f$. Eurydice, 2001: 192). Nous finirons donc ce trop court exposé sur les politiques linguistiques européennes en signalant qu'Eurydice mentionne l'apprentissage précoce des langues parmi les nouvelles initiatives de promotion de l'enseignement des langues recensées dans le chapitre consacré aux actions de la Communauté européenne. ${ }^{2}$

\section{Un cas de figure: la France face à l'enseignement présecondaire des langues vivantes}

Il convient à présent d'illustrer tout ce qui précède par des données issues d'un cas particulier, celui de la France. Notre principale source est, encore une fois,

${ }^{2}$ Il faut ici citer la conférence qui a eu lieu au Luxembourg en 1997, pendant laquelle les ministres de l'éducation de l'Union européenne ont adopté la résolution $98 / \mathrm{C} / 1$ favorable à ce type d'apprentissage. Un an plus tard paraissait une publication recensant les projets existants et exposant les conditions d'un apprentissage linguistique précoce réussi (Blondin, Ch. et al. "Les Langues étrangères dès l'école maternelle ou primaire. Conditions et résultats". Pratiques pédagogiques. Louvain-la-Neuve: De Boeck Université; cité par Eurydice, 2001: 187). 
Eurydice; elle sera complétée par le discours du ministre de l'éducation français du 27 mars 2001 ainsi que par les données disponibles fin 2004 sur les sites Eduscol (www.eduscol.education.fr) et Vie publique (www.vie-publique.fr).

La France a connu depuis 1954 et jusqu'en 1989 diverses expériences locales d'enseignement précoce des langues vivantes visant avant tout les élèves de 8 à 11 ans, mais aussi ceux de 4 et 5 ans. Cependant, ces projets pilotes ne concernaient le plus souvent que deux langues étrangères (l'anglais et l'allemand, surtout en Alsace où l'enseignement de l'allemand est intégré depuis longtemps) et ils ne touchaient que 2 à $3 \%$ des enfants d'une classe d'âge. Les évaluations réalisées en 1974 et 1980 ont fait état d'effets très limités, dus essentiellement à l'absence de prise en compte réelle des acquis antérieurs par les enseignants du collège ainsi qu'au manque de continuité entre programmes et manuels du primaire et du collège.

Les diverses expérimentations menées en France de 1989 à 1992, suite à une circulaire relative à "l'expérimentation contrôlée d'une langue vivante étrangère à l'école primaire", ont débouché sur la mise en place progressive d'un "enseignement d'initiation à une langue étrangère", qui représente "une grande rupture culturelle par rapport à la tradition du système scolaire français" (Gaudemar, 2002: 4). Ainsi, à partir de 2000 , la situation tend à évoluer positivement, depuis qu'il a été décidé d'introduire entre 2001 et 2006 l'enseignement obligatoire d'une langue étrangère comme une discipline à part entière figurant au programme de l'école primaire ( $c f$. Eurydice, 2001: 67). Certes, la volonté politique ne suffit pas à transformer la réalité; cette volonté est cependant indispensable pour éviter que les progrès obtenus restent cantonnés à des initiatives ponctuelles, toujours sujettes au risque de disparaître du jour au lendemain. En 2001, cet enseignement de 2 à 3 heures par semaine - assuré soit par des professeurs du secondaire payés en heures supplémentaires, soit par des intervenants extérieurs agréés - était déjà en voie de généralisation pour les élèves de 9 et 10 ans. Par ailleurs, depuis 1991, l'éventail des langues tend à s'élargir; tandis que depuis 1995, dans le cadre du "Nouveau contrat pour l'école", les élèves de 7 ans reçoivent quinze minutes quotidiennes d'initiation aux langues étrangères à l'aide de cassettes audiovisuelles produites et diffusées par le ministère ( $c f$. Eurydice, 2001: 68-69).

En principe, à partir de 2005, les élèves devraient bénéficier de l'enseignement d'une langue étrangère dès 5 ans, de sorte qu'à l'entrée au collège ils puissent commencer l'apprentissage d'une seconde langue. En effet, le ministre de l'éducation a annoncé en 2000 la mise en œuvre d'un plan ambitieux relatif au développement de l'enseignement des langues vivantes de la maternelle à l'université, ${ }^{3}$ dont l'un des trois axes forts était d'amener tous les élèves à la

${ }^{3}$ Le détail de ce plan est consultable sur le site Eduscol: http:/www.eduscol.education.fr 
maîtrise de deux langues vivantes, tandis que les deux autres visaient à préserver la diversité des langues vivantes et à offrir un choix varié de parcours possibles. Pour atteindre cet objectif énoncé dans le premier axe, c'est-à-dire, la maîtrise de deux langues vivantes par tous les élèves, le plan mettait l'accent sur plusieurs orientations, dont deux concernent spécifiquement l'apprentissage présecondaire: d'une part, la généralisation de l'enseignement des langues vivantes à l'école primaire et, de l'autre, l'acquisition de connaissances à travers les langues. ${ }^{4}$ Il s'agissait en effet de profiter d'une période où l'enfant est censé être particulièrement réceptif, et en postulant que plus l'apprentissage est précoce, plus vite l'élève sera apte à comprendre et à utiliser d'abord une, puis plusieurs langues différentes de sa langue nationale. Il s'agissait aussi d'affirmer la conviction que la langue est un outil de communication mais aussi un outil de construction des savoirs; par conséquent, elle doit non seulement être apprise, mais encore utilisée autant que possible.

Concrètement, ce plan prévoyait la généralisation immédiate de l'enseignement des langues vivantes aux enfants de 9 et 10 ans et son extension successive à ceux de 8 ans en 2002, à ceux de 7 ans en 2003, à ceux de 6 ans en 2004 et à ceux de 5 ans en 2005, pour débuter une deuxième langue à 11 ans. Cependant, on ne peut manquer de remarquer l'utilisation récurrente de l'imparfait dans toutes les références relatives au plan annoncé en 2000 par Jack Lang et soutenu encore avec force en 2001. En effet, face aux difficultés d'application du plan, qui concernent surtout la formation et le recrutement des maîtres, son successeur Luc Ferry a annoncé en 2002 que l'objectif était de mettre en place cet enseignement de la façon la plus large possible pour les enfants de 8 ans, avec une mise en place complète en CE1 au plus tard à la rentrée 2005. Comme le disait déjà en 2000 Maryline Baumard, "en matière de langues vivantes, l'école primaire court derrière les ministres" (Baumard, 2000: 53).

\section{L'écart entre les politiques officielles et la réalité sur le terrain}

Il faut à présent abandonner le terrain des politiques abstraites et se pencher sur les réalités du terrain. La généralisation de l'enseignement des langues vivantes à l'école primaire, qui est en soi un objectif difficilement critiquable, implique

${ }^{4}$ Nous évoquons ici essentiellement l'enseignement d'une ou plusieurs langues étrangères dans les situations les plus courantes de scolarisation au niveau primaire, en laissant donc de côté, à regret, le cas spécifique des "sections européennes", où la langue étrangère ne fait pas partie du cursus en tant que telle mais y joue un rôle primordial en tant que langue d'enseignement. Le plan mis en place en 2000 y fait quant à lui explicitement référence. 
un certain nombre de difficultés. Face à la demande accrue d'enseignants au niveau primaire, on a assisté le plus souvent "au règne du bricolage" (Baumard, 2000: 52), car les efforts se sont concentrés sur le quantitatif, tandis que les établissements peinent à prendre en compte le qualitatif. L'enseignement des langues vivantes a été largement assuré par des vacataires extérieurs souvent difficiles à recruter, notamment en zones rurales, malgré des exigences relativement faibles au niveau linguistique et pédagogique.

Or, paradoxalement, face à un objet d'enseignement de plus en plus complexe, la tendance s'oriente vers des enseignants de plus en plus qualifiés, tandis que les programmes d'études s'ouvrent à d'innovations et que la formation continue, facteur clé dans la mise en place des innovations méthodologiques, devient de plus en plus diversifiée et dynamique, car la formation initiale de la majorité des enseignants en service remonte à plus de vingt ans.

À terme, l'enseignement des langues vivantes doit être pris en charge par la majorité des enseignants du premier degré. Cela suppose la mobilisation et la formation des maitres actuellement en poste, le développement des échanges, la création de postes spécifiques, le recours à des maîtres itinérants; cela exige aussi de reconsidérer la formation initiale des nouveaux professeurs des écoles, en obligeant tous les candidats au concours à être titulaires d'un certificat en langue ( $c f$. www.vie-publique.fr).

La formation et le recrutement des enseignants compétents constituent les deux points les plus sensibles, mais il y en a d'autres. Maryline Baumard (2000:53) souligne que l'ambitieux projet de 2000 a éveillé des attentes parfois excessives des parents, qui confondent souvent enseignement d'une langue vivante et garantie d'une maîtrise totale de cette langue. Or, en évoquant la compétence plurilingue et pluriculturelle, le Cadre ne manque pas d'observer que "toute connaissance d'une langue est partielle, si apparemment 'maternelle' et 'native' soit-elle" (Conseil de l'Europe, 2001: 130).

Par ailleurs, "Depuis des décennies, chaque langue étrangère avait défini des attentes pour sa discipline sans se soucier des autres langues" (Baumard, 2003: 49). Dans une Europe où le maître mot n'est plus le bilinguisme mais le plurilinguisme, il faut désormais imaginer les mécanismes permettant de mettre en place un véritable travail commun à tous les acteurs du domaine des langues vivantes.

De son côté, Eurydice évoque les difficultés qu'impliquent la domination de l'anglais dans le choix des élèves et des familles; l'écart entre l'éventail des langues étrangères offertes aux élèves selon les documents officiels et celui réellement proposé par les établissements en raison de l'obligation d'effectif minimum, de la demande des parents ou de la force de la tradition; le besoin de s'assurer que les nouvelles approches didactiques préconisées puissent réelle- 
ment être appliquées dans les classes; ainsi que les adaptations nécessaires de l'organisation de l'enseignement proprement dite ( $c f$. Eurydice, 2001: 3; 96).

\section{Des pistes de réflexion sur la perspective mexicaine}

La liste des écueils pourrait s'allonger encore et encore; et l'écart persistera toujours entre les déclarations et les attentes institutionnelles et la réalité des classes. Cependant, ces orientations politiques sont en train de changer progressivement et surtout radicalement la perspective des langues étrangères en Europe. Elles ont déjà et continueront d'avoir des répercussions directes et indirectes sur l'enseignement/apprentissage des langues partout dans le monde, et donc au Mexique, par le biais des changements introduits dans la conception du matériel pédagogique, dans l'évaluation des compétences, dans les prérequis exigés aux étudiants étrangers, dans les certifications professionnelles, dans les programmes universitaíres d'échange et d'éducation à distance... Il revient à chacun d'articuler ce qui vient d'être exposé et sa situation spécifique. Nous souhaiterions néanmoins, pour finir, poser pêle-mêle sur la table un certain nombre d'interrogations, relatives à notre perspective locale: ${ }^{5}$

-Apprendre des langues, oui, mais quelles langues et pourquoi faire? Cette interrogation ne concerne pas exclusivement les langues étrangères, mais aussi toutes les langues minoritaires qui font la richesse linguistique de notre pays. Le Mexique est en effet un pays multilingue, dont de nombreux habitants sont bilingues voire plurilingues; or, ce phénomène ne concerne pas en priorité les enfants des écoles privées apprenant une langue étrangère dans un contexte scolaire comme un élément de distinction sociale, mais plutôt les enfants scolarisés en espagnol et qui n'ont pas l'espagnol pour langue maternelle.

-Comment défendre l'importance de l'apprentissage des langues vivantes -dans un contexte institutionnel où même des disciplines telles que la géographie et l'histoire ont récemment été remises en cause-, sans pour autant tomber dans le piège de la défense à outrance des motivations instrumentales? En fin de compte, il s'agit de débattre autour des finalités accordées à la scolarisation de nos enfants.

— Vu le poids du secteur privé aux niveaux préprimaire et primaire, du moins en ce qui concerne les classes aisées mexicaines, comment contribuer à enrichir la perspective de l'enseignement/apprentissage des langues dans le domaine de l'éducation publique?

- Comment enrichir le débat international autour de l'apprentissage précoce des langues en milieu scolaire à partir de notre expérience nationale?

${ }^{5}$ Ces interrogations sont largement inspirées du dossier déjà cité (Gaudemar, 2002). 
- Comment aboutir à une offre de langues continue et cohérente tout au long du parcours scolaire, qui permette de voir dans chaque langue étrangère un véritable outil de communication et de construction des savoirs et non un simple bibelot de luxe? Comment contribuer en fait à mettre en place puis à assurer la continuité des bonnes politiques linguistiques dans le milieu scolaire mexicain, voire des bonnes politiques éducatives tout court?

- Comment mettre l'accent sur la qualité de l'enseignement, et donc comment mettre en ouvre des formations initiales et continues capables de répondre aux défis actuels, dans un contexte où il existe très peu d'options de formation à l'enseignement précoce des langues étrangères et très peu de perspectives d'études de troisième cycle dans ce domaine? Comment exploiter au maximum les programmes universitaires et non universitaires d'échange existants, comment en concevoir d'autres de mieux en mieux adaptés à nos besoins et à nos attentes?

-Doit-on se contenter de former des enseignants pour mettre en pratique des orientations extérieures ou faut-il s'efforcer de consolider les possibilités de formation de chercheurs, voire de créer de nouveaux parcours de formation supérieure?

-Quelle(s) formation(s) spécifique(s) envisager pour les experts et les décideurs chargés de concevoir les politiques linguistiques nationales?

-Le plurilinguisme positif serait-il un luxe de pays riche? Un modèle scolaire "interculturel, axé sur le respect mutuel, l'éveil à d'autres cultures et les compétences transculturelles" (cf. Eurydice, 2001: 192) est-il à bannir sous prétexte d'utopie? Autrement dit, bien que la situation régionale multilingue de l'Europe diverge profondément de la situation latino-américaine, pouvons-nous envisager le plurilinguisme comme une priorité de nos pays?

- Si nous répondons positivement à la question précédente, comment contribuer depuis la perspective francophone à défendre le plurilinguisme et le pluriculturalisme, et donc l'espagnol et la diversité des manifestations culturelles hispanophones?

- Comment prendre en compte le chemin parcouru par les diverses instances responsables des politiques linguistiques européennes sans tomber dans la tentation d'intégrer aveuglément des idées conçues depuis un contexte bien spécifique tel que celui de l'Union européenne? Certes, les diverses réalisations du Conseil de l'Europe sont devenues des outils de référence obligée, mais ils restent nécessairement perfectibles et exigent d'être adaptés à notre réalité.

Nous le voyons, la question du bilinguisme s'inscrit de plain-pied dans le terrain du politique, et ce n'est pas par hasard que nous avons commencé aujourd'hui par évoquer cet aspect. Bien au-delà de sa dimension individuelle, le bilinguisme nous oblige à réfléchir sur notre société, sur les priorités qu'elle 
se donne, sur les valeurs qu'elle défend, sur le rôle qu'elle nous assigne. Bref, la question du bilinguisme nous oblige à mettre en perspective et à remettre en question notre rôle de véritables acteurs sociaux. Nous espérons avoir éveillé chez le lecteur l'envie d'explorer par lui-même un sujet qui concerne non seulement notre rôle professionnel ou familial, mais aussi notre rôle en tant que citoyens.

Références citées

BAUMARD, Maryline. 2000. "Primaire: les langues étrangères enfin pour tous". Le Monde de l'éducation, 285. Octobre. 52-53.

. 2003. "Langues vivantes: l'accent sur la culture". Le Monde de l'éducation, 311. Février. 49-51.

CONSEIL DE L'EUROPE. 2001. Cadre européen commun de référence pour les langues. Apprendre, enseigner, évaluer. Paris: Conseil de l'Europe/Didier.

Ouvrage disponible sur le site du Conseil de l'Europe: http:// www.coe.int EURYDICE. 2001. L'Enseignement des langues étrangères en milieu scolaire en Europe. Bruxelles: Eurydice (Les Études d'Eurydice).

Ouvrage disponible sur le site Eurydice: http://eurydice.org GAUDEMAR, Jean-Paul de. 2002. "Apprendre des langues étrangères: quelles langues et pourquoi faire". s. 1.: Desco.

Dossier accessible sur le site Eduscol: http://www.eduscol.education.fr www.vie-publique. fr 